\title{
Understanding neuronal dysfunction and loss in neurodegenerative disease
}

\author{
Flaviano Giorgini
}

Published online: 15 May 2013

(C) Springer-Verlag Berlin Heidelberg 2013

Recent advances in understanding the mechanisms underlying neurodegenerative disease have pushed the boundaries of potential therapeutics for these disorders. Patients suffering from Alzheimer's disease (AD), Parkinson's disease (PD), Huntington's disease (HD), and other neurodegenerative conditions exhibit a myriad of cognitive, motor, and psychological symptoms deriving from dysfunction and loss of specific populations of neurons in the brain. As age is the primary risk factor for all of these disorders, the increasing aging population is exhibiting greater numbers of affected individuals. In addition to the devastation these diseases afflict on patients and their families, such conditions place a growing economic burden on society. This issue of the Journal of Molecular Medicine contains six timely reviews from experts in the field, which cover recent advances on a broad array of mechanisms that have been implicated in the pathogenesis of neurodegenerative disorders.

Lotz and Legleiter [1] review the causative role of amyloidogenic protein oligomerization in neurodegenerative disease onset and progression. Amyloids are fibrillar protein aggregates that have been historically associated with the cellular toxicity observed in several neurodegenerative diseases. Evidence in recent years suggests, however, that intermediates to fibrils known as oligomers may play a central role in the neurotoxicity observed in these diseases. As oligomer formation is an early step in the pathogenesis of several neurodegenerative disorders, targeting this process may provide a method of treating these disorders by pre-empting downstream pathogenic cellular dysfunction. Furthermore, as conformational similarities exist between aggregates formed from different amyloidogenic proteins, related therapeutic strategies targeting oligomerization may emerge for diverse neurodegenerative diseases.

F. Giorgini $(\bowtie)$

Department of Genetics, University of Leicester, University Road, Leicester LE1 7RH, UK

e-mail: fg36@le.ac.uk
Mitochondrial dysfunction has long been closely linked to the pathogenesis of neurodegenerative disease. Lehmann and Martins [2] discuss the critical role of these cellular organelles in meeting the energy demands of neurons and how impaired mitochondrial function contributes to PD pathogenesis. The regulation of mitochondrial stress pathways and the importance of mitochondrial quality control in maintaining mitochondrial health are explored, as are the implications of these processes on neurodegenerative disease.

Träger and Tabrizi [3] provide a detailed overview of peripheral inflammation in neurodegenerative disease and highlight novel work exploring this process. While neuroinflammation is a well-studied aspect of neurodegenerative disorders, until recent years immune dysfunction in the periphery - and its contribution to pathogenesis - has been largely ignored. As peripheral inflammation appears to influence neurodegenerative disease progression and pathology, it is possible that treating this inflammation will have therapeutic relevance. The ready access to peripheral immune cells from patients' blood suggests that these cells may also provide insight into monitoring disease onset and progression.

Most research on the pathogenesis of HD and other polyglutamine disorders has focused on the causative, toxic nature of mutant proteins with expanded polyglutamine tracts and the downstream consequences. However, the expanded CAG repeats present in mutant transcripts have also been shown to be toxic and likely produce an additional cellular insult. Fiszer and Krzyzosiak [4] examine recent work exploring the contribution of RNA toxicity to the neuronal dysfunction and degeneration observed in these diseases. The basic mechanisms underlying RNA toxicity-as well as the experimental systems used for these studies-are also reviewed.

Eisbach and Outeiro [5] review recent advances in the understanding of the interplay between the protein $\alpha$-synuclein and intracellular trafficking in the context of PD. $\alpha$-Synuclein is the primary component of Lewy 
bodies - intracellular protein deposits which are a significant hallmark of PD pathology — and has also been linked to early onset familial cases of PD. $\alpha$-Synuclein likely contributes to perturbations in intracellular trafficking, which lead to neuronal dysfunction and, ultimately, death of these cells. The interactions of $\alpha$-synuclein with this trafficking machinery and the relevance of these to disease pathogenesis are discussed.

Finally, in Amaral et al. [6], we provide an overview of the contribution of the kynurenine pathway of tryptophan degradation to neurodegeneration and discuss the therapeutic potential of kynurenine 3-monoxygenase - as well as other enzymes in the pathway-for treating these disorders. The kynurenine pathway contains several neuroactive metabolites which are perturbed in a number of neurodegenerative diseases, including $\mathrm{AD}$ and HD. Recent advances using several animal models suggest that targeting this pathway either pharmacologically or genetically normalizes levels of these metabolites and is protective in $\mathrm{HD}$ and AD, and will likely have relevance in other neurodegenerative diseases.

In total, these review articles cover a wide array of cellular processes and mechanisms that contribute to the onset, progression, and pathology of neurodegenerative diseases. While the initiating insult or "trigger" in these diseases varies - and genetic and environmental factors have differing impacts - there are many commonalities in the pathogenesis of these disorders. Thus, novel insights into the pathology or treatment strategies for one of these diseases may have relevance for neurodegenerative conditions in general. Finally, as modulation of several different pathways can ameliorate phenotypes associated with disease, it is likely that future therapeutic interventions will consist of combinations of drugs which improve several different aspects of cellular and organismal dysfunction.

\section{References}

1. Lotz GP, Legleiter J (2013) The role of amyloidogenic protein oligomerization in neurodegenerative disease. J Mol Med. doi:10.1007/ s00109-013-1025-1

2. Lehmann S, Martins LM (2013) Insights into mitochondrial quality control pathways and Parkinson's disease. J Mol Med. doi:10.1007/ s00109-013-1044-y

3. Träger U, Tabrizi SJ (2013) Peripheral inflammation in neurodegeneration. J Mol Med. doi:10.1007/s00109-013-1026-0

4. Fiszer A, Krzyzosiak WJ (2013) RNA toxicity in polyglutamine disorders: concepts, models, and progress of research. J Mol Med. doi:10.1007/s00109-013-1016-2

5. Eisbach SE, Outeiro TF (2013) alpha-Synuclein and intracellular trafficking: impact on the spreading of Parkinson's disease pathology. J Mol Med. doi:10.1007/s00109-013-1038-9

6. Amaral M, Outeiro TF, Scrutton NS, Giorgini F (2013) The causative role and therapeutic potential of the kynurenine pathway in neurodegenerative disease. J Mol Med. doi:10.1007/ s00109-013-1046-9 